\title{
Antiretroviral compounds affect the granule-dependent mechanisms of lysis in CD8 $\mathrm{T}$ cells
} Anita Parmigiani*, Daria Trabattoni and Mario Clerici

Address: Department of Immunology, University of Milano, Milano, Italy

* Corresponding author

from 2006 International Meeting of The Institute of Human Virology

Baltimore, USA. 17-21 November, 2006

Published: 21 December 2006

Retrovirology 2006, 3(Suppl I):P49 doi:I0.II86/I742-4690-3-SI-P49

(C) 2006 Parmigiani et al; licensee BioMed Central Ltd.

\section{Background}

Cytotoxic T-lymphocytes (CTLs) are essential for suppression of viral replication and, in particular, they have a pivotal role in control of progression of HIV infection. It has been demonstrated that HIV-specific CTL responses are defective in HIV-infected patients undergoing highly active antiretroviral therapy (HAART). In this study we investigated the effects of antiretroviral compounds on the granule-dependent mechanisms of lysis in peripheral blood mononuclear cells (PBMCs).

\section{Materials and methods}

PBMCs of 10 HCs were incubated with 3 different antiretroviral drugs combinations: combination A: AZT (NRTI) + 3TC (NRTI) + IDV (PI); combination B: d4T (NRTI) + ddI $(\mathrm{NRTI})+\mathrm{NFV}(\mathrm{PI})$; combination C: 3TC (NRTI) + EFV (NNRTI) + TDF (NRTI). To evaluate the CTLs function we measured: production and release of granule-dependent effector molecules (perforin, granzyme B); production and release of granule-independent effector molecules (IFN-gamma, TNF-alpha); degranulation markers (LAMP1 and LAMP2). To assest the immunomodulant effects of IL-15, PBMCs were also incubate in presence of this cytokyne.

\section{Results}

Antiretroviral compounds reduce the granzyme B and perforin production (while they don't affect the IFN-gamma and TNF-alpha production). Moreover, one of the 3 tested combinations of antiretroviral compounds (combination $B)$ reduces the granzyme $B$ release and affects the degran- ulation in CTLs. IL-15 increases the levels of granzyme B and perforin.

\section{Conclusion}

Antiretroviral compounds mainly affect the expression of genes encoding for Pfp and GranzB, and deteriorate the mechanism of degranulation in CD8+ $\mathrm{T}$ cells. $\mathrm{L}-15$ restores both the granule-dependent and the granuleindependent cytotoxic mechanisms. Basd on these data, IL-15 seems to be useful in overcoming the negative effects of antiretroviral compounds on the cytotoxic function. 